\title{
Vibrational Properties and Stabilization Mechanism of the Doped GeTe Phase Change Material Amorphous Phase
}

\author{
Jean-Yves Raty* \\ Physics of Solids, Interfaces and Nanostructures, B5, \\ University of Liège, B4000 Sart-Tilman, Belgium \\ Pierre Noé, Giada Ghezzi, and Sylvain Maîtrejean \\ CEA, LETI, MINATEC Campus, 17 rue des Martyrs, 38054 Grenoble Cedex 9, France. \\ Christophe Bichara \\ CNRS-CINAM , Campus de Luminy - Case 913 F13288 - Marseille - Cedex 9 - France \\ Françoise Hippert \\ LMGP, CNRS Grenoble-INP, MINATEC, 3 parvis Louis Néel, \\ BP 166, 38016 Grenoble Cedex 1, France and \\ LNCMI, CNRS UJF UPS INSA, 25 rue des Martyrs , \\ BP 257, 38042 Grenoble Cedex 9, France
}

\begin{abstract}
Doping Chalcogenide Phase Change Materials was shown to improve the stability of the amorphous phase at high temperature and to strongly increase the crystallization temperature. In this work, we use ab initio molecular dynamics together with Fourier Transform InfraRed (FTIR) spectroscopy to address the stabilization of GeTe doped with Nitrogen and Carbon in the amorphous phase. The comparison between the simulation and experimental results allows in depth understanding of the mechanisms. The inclusion of $\mathrm{C}$ and $\mathrm{N}$ leads to an increase in high frequency vibrational modes and to a lowering of the Boson peak intensity. The reduction of the density of floppy vibrational modes and the computed increase of the mechanical rigidity are responsible for the higher activation energy for crystallization. The mechanism described here could apply more generally to stabilize other Ge-Sb-Te phase change materials and iono-covalent glasses at high temperature.
\end{abstract}


Chalcogenide Phase Change Materials (PCMs) such as Ge-Sb-Te based alloys (GST) show a unique ensemble of properties. They exhibit a reversible and extremely fast (ns) phase change between an amorphous and a crystalline phase (a-phase and c-phase) that have quite different electronic properties [1]. The a-phase is characterized by a high resistivity and low optical reflectivity while the c-phase by a much lower resistivity and higher reflectivity. From the point of view of the atomic structure, these phase change materials appear somehow paradoxical. Indeed, the fast recrystallization suggests that atomic rearrangements are of little importance, but the strong properties contrast implies very different structures. Many experiments and simulations have helped understanding these properties especially regarding the structure of the amorphous phase. Ab initio Molecular Dynamics (AIMD) simulations [2-8] in agreement with various X-ray absorption and diffraction measurements [9-12] showed that the structures of all amorphous GSTs, including GeTe, are rather similar from the point of view of the atomic local order. Especially, Ge atoms have been shown to be either $\mathrm{sp}^{3}$ bonded in a tetrahedral environment or p-bonded, in a distorted octahedral environment, the fraction of $\mathrm{sp}^{3}$-bonded Ge ranging typically below $30 \%$.

The applications of PCMs for data storage have attracted much interest. Their optical contrast has been used since long in DVDs while their resistive contrast makes them currently one of the most promising alternative technologies for non-volatile resistive memories commonly called PCRAM (Phase Change Random Access Memories). However, to fulfill the challenges of PCRAMs technologies, it is necessary to improve some of the PCMs properties, mainly to reduce the drift phenomenon (time dependence of the amorphous state resistivity) and to increase the retention time at higher operational temperatures for embedded applications. These improvements imply a more stable amorphous phase, without alteration of the unique properties that made the PCM field promising, such as their fast switching ability, a low reset current for the transition from the crystalline to the amorphous state in devices and a strong contrast in properties between the c- and a- phases. These needs have triggered the search for new, improved compositions, extending away from the usual Ge-Sb-Te (GST) ternary compounds. An alternative promising way to improve PCMs is the inclusion of proper dopants at high concentration, such as $\mathrm{C}$ or $\mathrm{N}$ [13]. Many works have been done on doped GST [14]. Here we focus on the effect of doping in GeTe. Indeed, undoped GeTe has better retention properties than GST [15]. Moreover it has been shown recently that doping GeTe with $\mathrm{C}$ or $\mathrm{N}$ leads to a major increase of the crystallization tem- 
perature $T_{\mathrm{x}}$ and to an improved data retention while allowing at the same time for a lower reset current $[15,16]$. Compared to pure GeTe with a $\mathrm{T}_{\mathrm{x}}$ of about $180^{\circ} \mathrm{C}$, the incorporation of $10 \mathrm{at} \% \mathrm{~N}$ or $\mathrm{C}$ atoms (hyper-doping) increases $\mathrm{T}_{\mathrm{x}}$ up to $264^{\circ} \mathrm{C}$ and $317^{\circ} \mathrm{C}$ respectively. The magnitude of these effects and the fact that GeTe is a simpler binary alloy as compared to GSTs, makes the doping of GeTe a case study to understand not only the origin of these effects but more generally, how compositional changes relate with the stability of glasses.

As the phase change is associated to modifications of the local atomic arrangement, it is first necessary to obtain structural information about the amorphous phase. In a recent paper we described the structure of the C-doped GeTe a-phase [17]. It was shown that carbon deeply modifies the structure of the amorphous phase through long carbon chains and tetrahedral and triangular units centered on carbon. In the simulations $\mathrm{C}$ atoms have been found to be bound to both Ge and Te. In the case of N-doped GeTe, recent studies have shown that $\mathrm{N}$ is predominantly bonded to Ge atoms in as-deposited amorphous films $[18,19]$. To our knowledge, no AIMD simulations have been performed on N-doped GeTe so far. On the other hand, information about the dynamics of the amorphous is essential to get insight on its stability, as it has been shown in the general case of glasses [20-22].

In this article, we present a combined theoretical (AIMD) and experimental (FourierTransformed InfraRed, FTIR, spectroscopy) study of the vibrational properties of C and N doped GeTe. Strong evolutions are observed that allow us to understand the origin of the stability induced by doping, which is shown to be related to the evolution of the Boson peak usually observed in other glasses.

Doped and undoped GeTe $150 \mathrm{~nm}$ thick films were deposited by magnetron sputtering in an Ar atmosphere on $200 \mathrm{~mm}$ Si (100) wafers. N-doped samples were prepared by reactive sputtering of a GeTe target using an $\mathrm{Ar} / \mathrm{N}_{2}$ gas mixture. C-doped samples were prepared by co-sputtering of GeTe and C targets. The compositions of the samples were varied by changing the $\mathrm{Ar} / \mathrm{N}_{2}$ ratio in case of $\mathrm{N}$-doped samples and by changing the sputtering power applied to the GeTe and C targets for the C-doped GeTe. The Ge and Te concentrations in the films were measured by Rutherford Back Scattering (RBS) and the $\mathrm{N}$ and $\mathrm{C}$ dopant concentrations by Nuclear Reaction Analysis (NRA). Four different C and N dopant concentrations of $5,10,15$ and 20 at\% with an accuracy of \pm 1 at\% were deposited. A pure GeTe thin layer has also been deposited as a non-doped reference sample. In all the studied samples, there is a small excess of Ge (corresponding to a Ge/Te ratio 52/48). The thick- 
nesses of the deposited layers and the deposition homogeneity on the $200 \mathrm{~mm}$ Si substrates are controlled by X-Ray Reflectivity (XRR). The reflectivity measurements were carried out by monitoring the reflectivity of the PCMs samples under Ar atmosphere with a laser probe at $632 \mathrm{~nm}$ as a function of the temperature applied to the samples by a heating chuck with a controlled heating rate of $10^{\circ} \mathrm{C} / \mathrm{min}$. The amorphous phase is characterized by a lower reflectivity than the crystalline phase. Thus the phase transformation is characterized by a significant increase of the measured reflectivity value as shown in Fig. 1. We can also define the crystallization temperature $T_{\mathrm{x}}$ as the point of maximum derivative of the measured reflectivity curve.
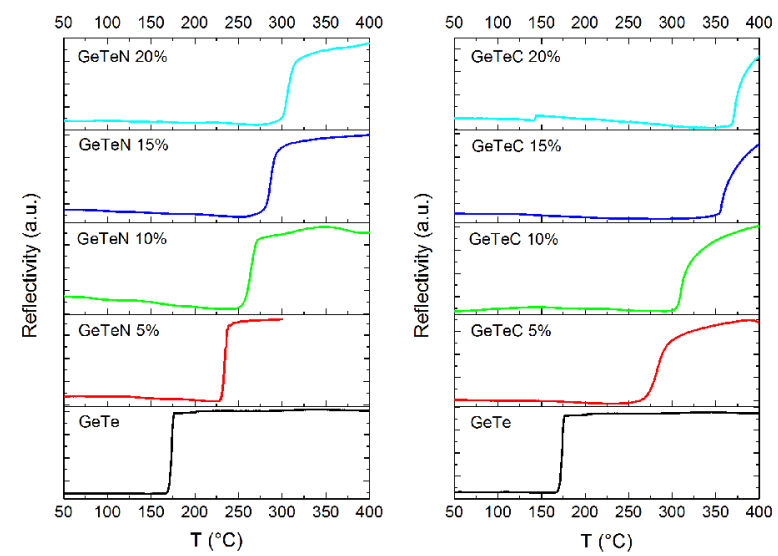

Figure 1: Reflectivity curves for C- (left panel) and N- (right panel) doped GeTe measured upon heating at a rate of $10^{\circ} \mathrm{C} /$ minute. These curves show the optical contrast observed upon crystallization, as well as the increase of the crystallization temperature upon doping.

FTIR absorbance spectra of the samples were collected in transmission mode using BIORAD QS500 and Bruker VERTEX 80V FTIR spectrometers in order to cover the range from 100 to $4000 \mathrm{~cm}^{-1}$. Prior to deposition the absorbance spectra of the silicon wafers have been measured. These blank spectra have been subtracted from the samples spectra in order to remove the contribution of $\mathrm{Si}$ phonons modes and that of dissolved element such as $\mathrm{O}$ in silicon wafers. All spectra were recorded with a resolution of $2 \mathrm{~cm}^{-1}$ and averaged over 64 scans for the 400-4000 $\mathrm{cm}^{-1}$ range (High Frequency range), and at $4 \mathrm{~cm}^{-1}$ on 32 scans for the 100 to $400 \mathrm{~cm}^{-1}$ range (Low Frequency range).

The GeTe, C and N-doped GeTe structural models were generated using Ab Initio Molecular Dynamics. The details of the GeTe and C-doped GeTe with 16 at\% C structures are 
given in Ref. [17]. The 10 at\% N doped model was generated in the same manner with a 21 N, 97 Ge, 92 Te atoms system to match the experimental Ge/Te atomic ratio (52/48). The electronic properties were computed within density functional theory (DFT) using PAW potentials and the PBE-GGA exchange correlation. The wavefunctions were expanded on a planewaves basis up to a $325 \mathrm{eV}$ cutoff energy. Constant volume molecular dynamics together with a Nose thermostat was then used to simulate the liquid structure at $3000 \mathrm{~K}$ for $10 \mathrm{ps}$ at the GeTe liquid density. The system was then equilibrated at $1000 \mathrm{~K}$ for 10 ps, with a progressive change in cell size to ensure a negligible stress and then cooled down to ambient temperature with a $5 \mathrm{~K} / \mathrm{ps}$ ramp, the cell size being reoptimized every $20 \mathrm{ps}$. The final models were then simulated for $40 \mathrm{ps}$ at $200 \mathrm{~K}$ without thermostat. The dynamical properties were computed using the NMoldyn code [23], the vibrational density of states (VDoS) being computed by Fourier transforming the van Hove autocorrelation function with a $0.3 \mathrm{THz}$ resolution.

The FTIR absorbance spectra of undoped and C and N-doped GeTe samples are plotted in Fig.2 and Fig.3. Let us first discuss the high frequency range above $12 \mathrm{THz}\left(400 \mathrm{~cm}^{-1}\right)$. In this range the pure GeTe sample has no absorption at all whereas a strong absorption peak is present around $18.6 \mathrm{THz}$ in C-doped samples (slightly bimodal shape) and $21 \mathrm{THz}$ in N-doped samples. These new peaks, absent in GeTe, grow with increasing dopant concentration. Similar new vibrational modes are observed in the calculated VDoS of C- and N-doped GeTe in the 12 to $24 \mathrm{THz}$ frequency range (see Fig.3). In N-doped GeTe no modes are detected above $24 \mathrm{THz}$ neither in the absorbance spectra nor in the calculated VDoS. In C-doped GeTe a high frequency contribution to the FTIR absorbance spectra is observed at $54 \mathrm{THz}$. Its amplitude increases with the $\mathrm{C}$ content. A broad contribution is also detected around $42 \mathrm{THz}$ but only in the samples with the highest $\mathrm{C}$ content. Modes with frequencies larger than $24 \mathrm{THz}$ are also present in the VDoS of the C-doped GeTe model, and are associated to $\mathrm{C}-\mathrm{C}$ bonds, as we will show hereafter. In summary, the simulations reproduces well the experiment trends, given that a direct comparison of the amplitudes is meaningless (the simulation yields the VDoS while the FTIR absorbance depends on the nature of the vibration mode).

In the low frequency range (from $3 \mathrm{THz}$ to $12 \mathrm{THz}$, see Fig.2) clear trends in the FTIR spectra are also observed upon both $\mathrm{C}$ and $\mathrm{N}$ doping. The peak at $4.5 \mathrm{Thz}$ is gradually disappearing in favor of a higher frequency shoulder, above 7.5 Thz. The comparison with 


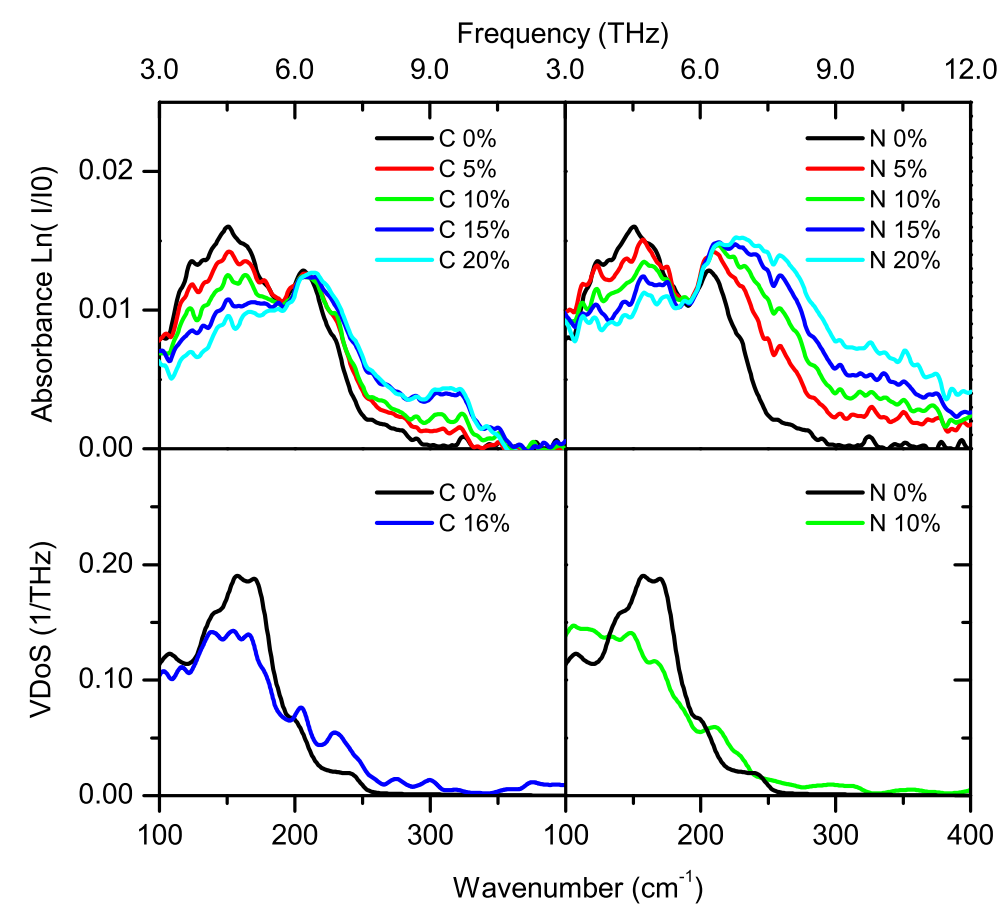

Figure 2: Experimental low frequency FTIR absorbance spectra of as-deposited undoped, C-doped and N-doped GeTe thin films (upper panels). The corresponding calculated vibrational densities of states (VDoS) are plotted on the lower panels. The VDoS of undoped GeTe is null above $8 \mathrm{THz}$.

the calculated VDoS shows a similar decrease of the $4.5 \mathrm{THz}$ peak with the additional information gathered from the species-projected VDoS that it comes from a decrease of Ge and Te associated vibrations, the ratio of the Ge and Te contributions, roughly equal to 2, to this peak being insensitive to doping (Fig.4). The shoulder detected in the $6-12 \mathrm{THz}$ range in FTIR spectra in the doped samples comes from the dopant themselves, as can be seen in Fig.5, since the maximum frequency of both the Te- and Ge-projected VDoS are the same (around $8 \mathrm{THz}$ ) in doped and undoped GeTe (Fig. 4). The main contributions to that shoulder arise from C-C bonds in C-doped GeTe and from 3-fold bonded nitrogen atoms in N-doped GeTe.

The details of the dopant site-projected VDoS are presented in Fig.5 and Fig.6. It can be seen that the new modes that are created between $12 \mathrm{Thz}$ and 24 Thz are essentially due to C-Ge and C-Te bonds for C-doped GeTe and to N-Ge only in N-doped GeTe, as expected from $\mathrm{C}$ and $\mathrm{N}$ small masses. As shown in [17], C-Ge and C-Te bonds are numerous in C-doped GeTe, though the GeTe skeleton of the structure remains similar to the undoped compound. It should be noticed that during the simulated annealing procedure, the initial 


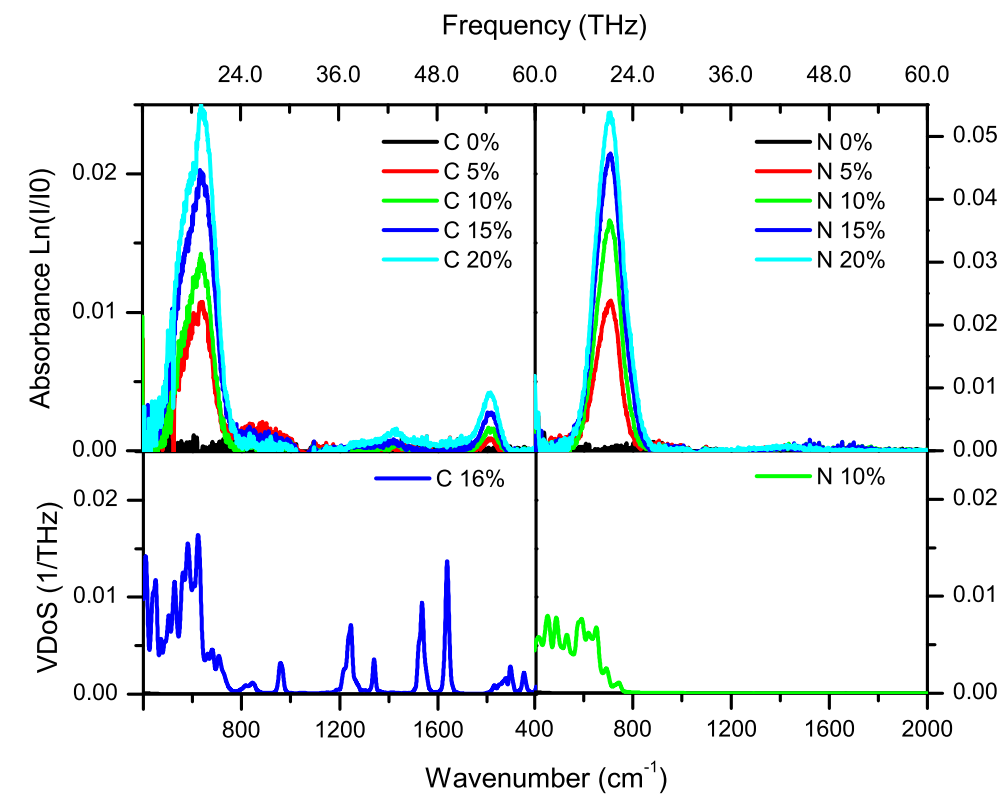

Figure 3: Experimental high frequency FTIR absorbance spectra of as-deposited undoped, C-doped and N-doped GeTe thin films (upper panels, different scales are used for the left and right panels). The FTIR spectrum of undoped GeTe is null in the high frequency range. The corresponding calculated vibrational densities of states (VDoS) are plotted on the lower panels.

configuration was taken from a liquid sample equilibrated at very high temperature (3000 $\mathrm{K})$, so that any kind of possible bond was present in the structure, including N-Te bonds. However, due to the low $\mathrm{N}$ concentration, no $\mathrm{N}_{2}$ molecule was present. It is thus remarkable upon annealing, all N-Te bonds have been disrupted, and statistically unlikely $\mathrm{N}_{2}$ molecules have formed (involving $4 \mathrm{~N}$ atoms out of a total of 21). The other $\mathrm{N}$ atoms are either bound to 3 Ge atoms in a pyramidal geometry or tetrahedrally to 4 Ge atoms, with similar proportions. The $\mathrm{N}_{2}$ molecular vibrations are observed around $70 \mathrm{THz}$, though rather poorly sampled due to the relatively large time step used ( $3 \mathrm{fs}$ ). The duration of the simulation also allows for identifying the $\mathrm{N}_{2}$ rotational modes around 2.5 Thz. Note that the presence of $\mathrm{N}_{2}$ in $\mathrm{N}$-doped amorphous GeTe films has been found by X-ray absorption experiments in Ref. [19] but not in Ref.[18].

In the $\mathrm{C}$ doping case, the highest frequency modes are associated to $\mathrm{C}-\mathrm{C}$ bonds in various $\mathrm{sp}$ and $\mathrm{sp}^{2}$ environments. Due to the limited sampling of the configurations space and to the variable sensitivity to vibration modes in FTIR spectroscopy, these high frequency modes are spread between 24 and 70 Thz, while in the experiment (see Fig.3), they are limited to 


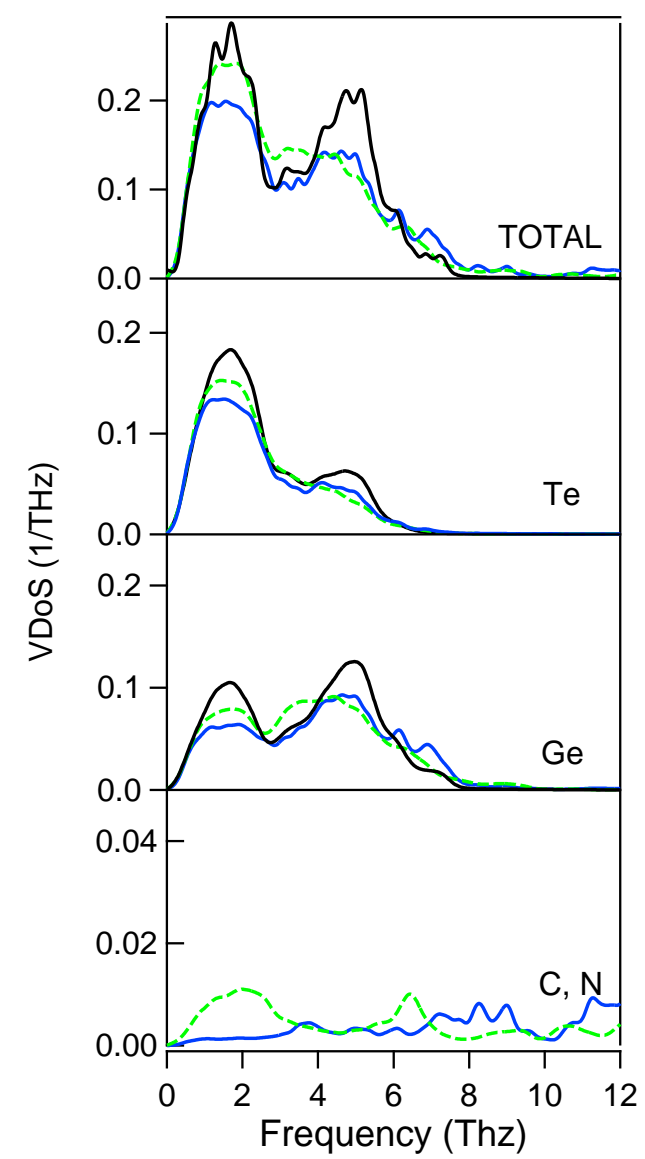

Figure 4: Total VDoS (normalized to unity) obtained from the AIMD trajectories (top panel). The total VDoSes are decomposed onto the different atomic species (lower panels): VDoS of GeTe, C-doped GeTe with 16at\% C and N-doped GeTe with 10at\% N are plotted with plain black, plain blue and dotted green lines, respectively.

two distinct frequency domains (around 42 and $54 \mathrm{Thz}$ ).

The simulation also indicates that the density of vibrational states strongly decreases in the low frequency range (below $3 \mathrm{THz}$ ), inaccessible to the experiment, upon doping (see Fig.7). The comparison with the VDoS of crystalline pure GeTe [24] reveals interesting trends: upon amorphization of pure GeTe, we observe the same elastic softening of the crystal acoustic modes that is usually observed in glasses [20], but also the hardening of the highest crystal optical modes that has been measured in GST [25]. The inclusion of dopants reinforces this vibrational hardening of the amorphous structure, but at the same time, due to normalization of the number of modes, the creation of higher frequency modes further depletes the density of modes at low frequency (0-3 Thz). This effect is stronger in C-doped 


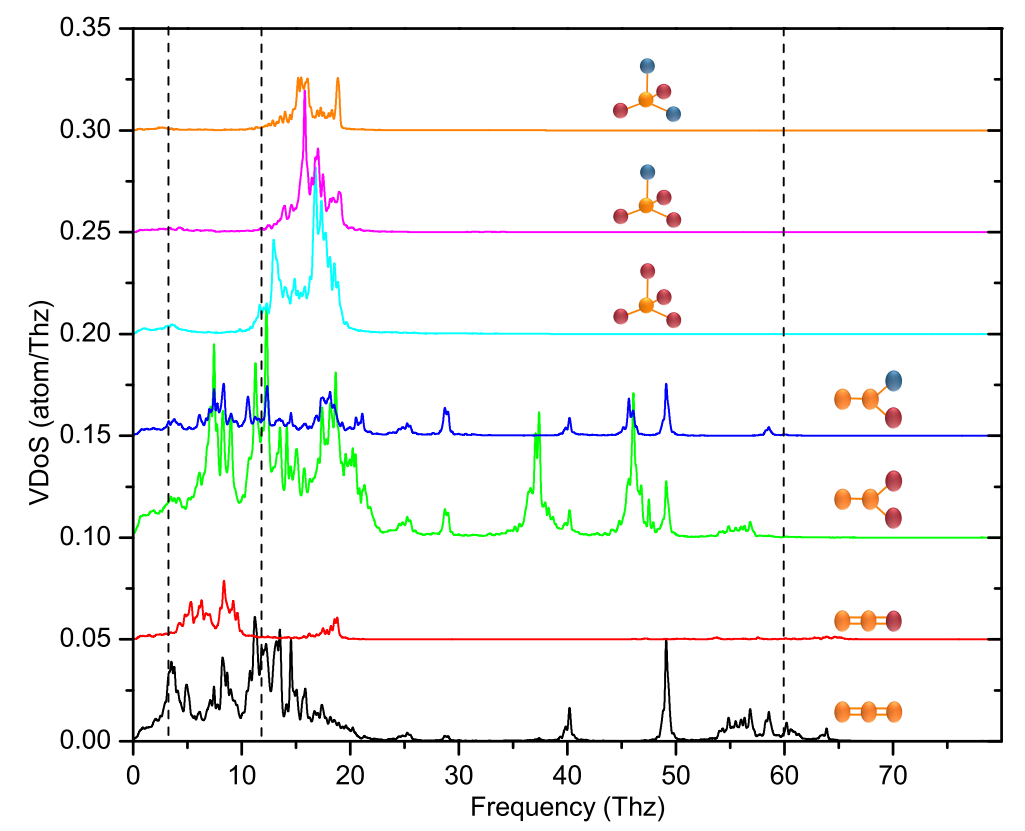

Figure 5: Dopant site-projected vibrational densities of states for the AIMD simulated C-doped GeTe with 16at\% C. Contributions have been summed up according to the local environment of the dopant atom (sketched on the graph). C, Ge and Te atoms are drawn in orange, red and blue, respectively. The dotted vertical lines separate the regions accessible to the different FTIR spectrometers.

GeTe as both the Ge and Te species are bound to $\mathrm{C}$ atoms, thus having their vibrational modes shifted to higher energy, while in N-doped GeTe only Ge centred vibrations are directly affected.

In order to further relate the structural and dynamical changes upon doping of GeTe to the enhanced stability of the amorphous phase at high temperature, we measured [26] the activation energy for crystallization, $\mathrm{E}_{\mathrm{a}}$ using the Kissinger method. $\mathrm{E}_{\mathrm{a}}$ values are equal to $2 \pm 0.1 \mathrm{eV}$ for GeTe, $3.9 \pm 0.2 \mathrm{eV}$ for N-doped GeTe with 10 at\% $\mathrm{N}$ and $4.52 \pm 0.2 \mathrm{eV}$ for C-doped GeTe with 16 at\% C.

As the crystallization process involves breaking bonds to create new local environments, it is sound to estimate how a local atomic environment is mechanically constrained by its surroundings. To quantify this degree of stress inside the models, we used the Maxwell constraints counting method described by Micoulaut et al. [27]. It consists of counting the rigid angles and distances between an atom and its neighbors. This measure of the number of effective constraints, $\mathrm{n}_{\mathrm{c}}$, is obtained through the analysis of the standard deviation of the 


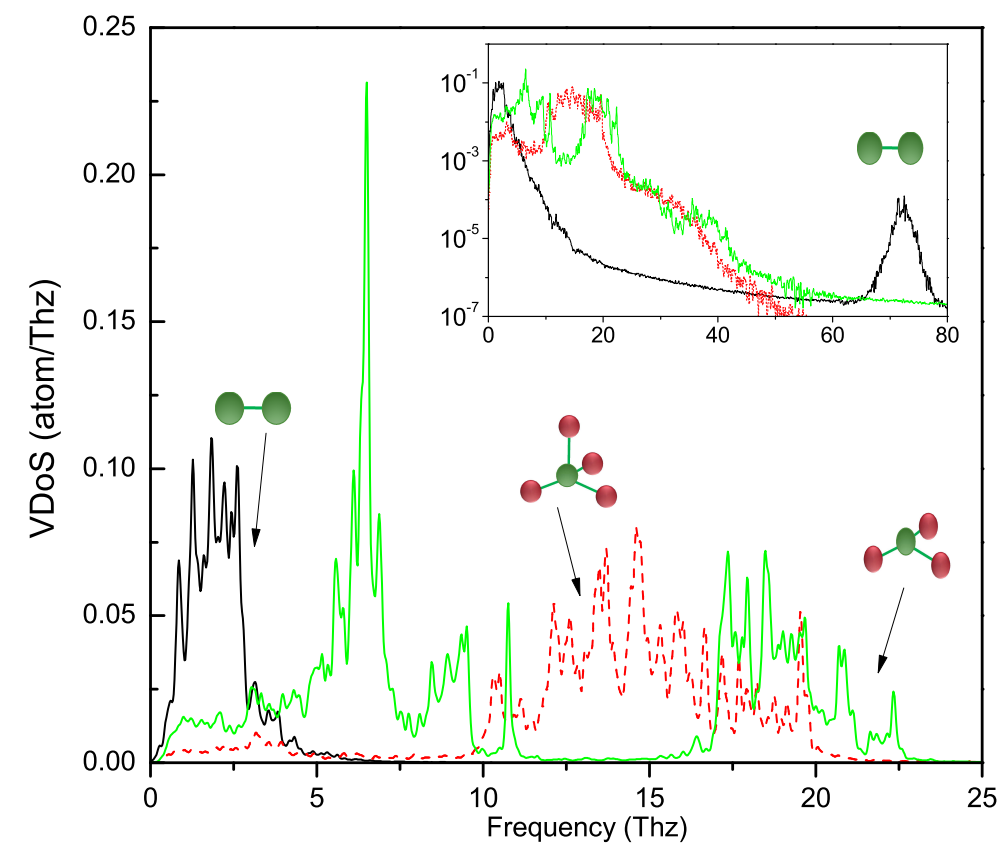

Figure 6: Dopant site-projected vibrational densities of states for the AIMD simulated N-doped GeTe with 10at\% N. N and Ge atoms are drawn in green and red, respectively. The arrows indicate the curves corresponding to the different kinds of atomic environments (molecular, pyramidal and tetrahedral). The insert shows the contribution of $\mathrm{N}_{2}$ vibrations at high frequency.

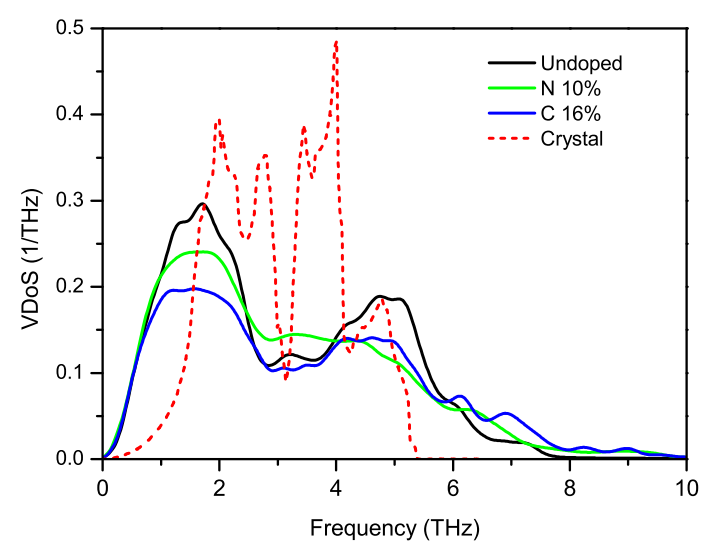

Figure 7: VDoS of amorphous GeTe, C-doped GeTe with 16 at\% C and N-doped GeTe with 10 at\% N, together with the computed crystalline GeTe phonon DoS (from Ref. [24]).

angles and neighbors distance distributions. The average value of constraints, $\mathrm{n}_{\mathrm{c}}$, is given by a sum over the different types of local environments of each atomic species using the formula $n_{c}=\sum_{i, j} c_{i} p_{i j}\left(B B_{i j}+B S_{i j}\right), c_{i}$ being the concentration of species $i, p_{i j}$ being the proportion of type $j$ local environment for species $i, B B_{i j}$ and $B S_{i j}$ being the corresponding number 
of bond bending and bond stretching mechanical constraints, respectively. We obtain $\mathrm{n}_{\mathrm{c}}$ values of 4.6, 4.8 and 5.0 for GeTe, N-doped GeTe with 10 at\% N and C-doped GeTe with 16 at\% C (see Table I), respectively. These values correlate well with increasing $\mathrm{E}_{\mathrm{a}}$ and $\mathrm{T}_{\mathrm{x}}$ , and indicate that the stressed rigid character of the GeTe glass is reinforced upon doping with $\mathrm{C}$ and $\mathrm{N}$, essentially because of the slight increase of the tetrahedral character of the Ge atoms.

On the other hand, the fact that the system has to reorganize more bonds is not the only relevant parameter to the crystallization process. The evolution of the VDoS peak below $3 \mathrm{THz}$ is also linked to the stability of the glass. This peak corresponds to the so-called Boson peak (BP) that is a characteristic of collective excitations in glasses. It was established recently that this BP is related to the first van Hove singularity of the corresponding crystal's TA modes (below 3-4 Thz in [20-22]). Indeed, for the GeTe crystal, the TA mode singularity is found at $1.55 \mathrm{THz}[24]$, which appears as a shoulder on the crystal VDoS and corresponds to the median frequency of the low frequency VDoS peak in the glass (Fig.7).

In the general case of glasses, it has been demonstrated that an increase in the BP height is the manifestation of a lesser glass stability [28]. This is due to the fact that these low frequency modes involve larger atomic root mean squared displacements [29]. On the other hand, both theory [30] and experiment [31] have shown that the BP height is increasing with the reduction of the number of mechanical constraints. Doping with $\mathrm{C}$ and $\mathrm{N}$ produces the inverse effect on a-GeTe: they increase the number of constraints and decrease the density of BP modes.

It thus appears that the stabilization of the GeTe glass upon doping occurs at two levels. First, the light elements used as dopant create stiffer, stronger bonds. Second, the mechanical constraints on the non dopant elements are increased upon doping (due to the slightly higher coordination number). As discussed before, these effects are directly reflected in the decrease of the BP. A similar behavior of the boson peak and lowest frequency modes has indeed been observed in silicate glasses [21] upon decreasing temperature, which gives further confirmation that the observed decrease in the lowest frequency VDoS upon doping is related to a higher activation energy and thus to an improved stability at high temperature.

In summary, the inclusion of $\mathrm{N}$ and $\mathrm{C}$ dopant elements in GeTe has a double effect: they create high frequency, localized modes, and at the same time decrease the density of low frequency acoustic-like modes (because of C-Ge, C-Te and N-Ge coupling), therefore 


\begin{tabular}{|c|c|c|c|c|c|}
\hline Compound:Species & $\mathrm{Z}$ & $\mathrm{p}$ & $\mathrm{BS}$ & $\mathrm{BB}$ & Contribution to $\mathrm{n}_{\mathrm{c}}$ \\
\hline \hline Ge97Te92N21:Ge & 4 & 0.43 & 2 & 5 & 1.38 \\
\hline & 3 & 0.57 & 1.5 & 3 & 1.18 \\
\hline Ge97Te92N21:Te & 3 & 0.75 & 1.5 & 3 & 1.48 \\
\hline & 2 & 0.25 & 1 & 1 & 0.22 \\
\hline Ge97Te92N21:N & 4 & 0.62 & 2 & 5 & 0.36 \\
\hline & 3 & 0.29 & 1.5 & 3 & 0.13 \\
\hline & 1 & 0.19 & - & - & 0 (free molecules) \\
\hline Ge92Te86C32:Ge & 4 & 0.59 & 2 & 5 & 1.82 \\
\hline & 3 & 0.41 & 1.5 & 3 & 0.81 \\
\hline Ge92Te86C32:Te & 3 & 0.88 & 1.5 & 3 & 1.62 \\
\hline & 2 & 0.12 & 1 & 1 & 0.1 \\
\hline Ge92Te86C32:C & 4 & 0.16 & 2 & 5 & 0.17 \\
\hline & 3 & 0.53 & 1.5 & 3 & 0.36 \\
\hline Ge105Te105:Te & 3 & 0.88 & 1.5 & 3 & 1.98 \\
\hline & 2 & 0.12 & 1 & 1 & 0.12 \\
\hline & 2 & 0.31 & 1 & 1 & 0.09 \\
\hline & 4 & 0.21 & 2 & 5 & 0.73 \\
\hline & 3 & 0.79 & 1.5 & 3 & 1.78 \\
\hline
\end{tabular}

Table I: Details of the calculations of the average number of constraints $n_{c}$ for all the model structures obtained with AIMD (method described in Ref.[27]). $\mathrm{Z}=$ coordination number, $\mathrm{p}=$ fraction of Z-fold coordinated atoms, $\mathrm{BB}=$ number of bond bending constraints, $\mathrm{BS}=$ number of bond stretching constraints, and contribution of the specific environment to the compound $\mathrm{n}_{\mathrm{c}}$ value. Distance cutoffs have been chosen equal to $2.93 \AA, 3.15 \AA, 2.43 \AA$ and $2.15 \AA$ for Ge, Te, N and C atoms, respectively (distances obtained from the decomposition of the partial g(r)'s - see Ref.[27]). The absolute values of $\mathrm{BS}+\mathrm{BB}$ are dependent on the choice of these cutoff distances, however the observed trends are robust (increasing number of constraints when going from GeTe to N-GeTe and $\mathrm{C}-\mathrm{GeTe})$. 
improving the stability of the glass. This diminished Boson peak goes with an increased mechanical rigidity which explains why these doped GeTe compounds have a higher recrystallization temperature than the undoped ones. This result suggests that an efficient way to improve data retention in amorphous PCMs in general could be achieved by doping with light elements that at the same time covalently link to the glass network and act as traps for high frequency vibrational modes. This stabilization mechanism with light dopants appears to be very general, and we expect that it could apply more generally to a vast range of iono-covalent glasses.

The authors wish to thank V. Sousa, L. Perniola, A. Persico, E. Henaff, M. Tessaire, A. Roule and E. Souchier at CEA-LETI and acknowledge the support from the French Nanosciences foundation (Percevall), the FRS-FNRS, an ARC grant ("Themoterm" 10/1503) and Wallonie-Bruxelles-International. Computer time was obtained trough the RI283493 EU-FP7 grant and the PRACE consortium.

* Electronic address: jyraty@ulg.ac.be

[1] M. Wuttig and N. Yamada, Nat. Mater. 6, 824 (2007).

[2] J.Y. Raty, C. Otjacques, J.P. Gaspard, and C. Bichara, Solid St. Sci. 12, 193 (2010).

[3] R. Mazzarello, S. Caravati, S. Angioletti-Uberti, M. Bernasconi, and M. Parrinello, Phys. Rev. Lett. 104, $085503(2010)$.

[4] J. Akola, J. Larrucea, and R. O. Jones, Phys. Rev. B 83, 094113 (2011); and references therein.

[5] J. Hegedüs, and S.R. Elliott, Nat. Mater 7(5), 399 (2008).

[6] T. H. Lee, and S.R. Elliott, Phys. Rev. Lett. 107, 145702 (2011).

[7] M. Krbal, A. V. Kolobov, P. Fons, J. Tominaga, S.R. Elliott, J. Hegedüs, and T. Uruga, Phys. Rev. B, 83, 054203 (2011).

[8] M. Xu, Y.Q. Cheng, H.W. Sheng, and E. Ma, Phys. Rev. Lett., 103, 195502 (2009).

[9] P. Jovari, I. Kaban, J. Steiner, B. Beuneu, A. Schöps, and M. Webb, Phys. Rev. B, 77, 035202(2008).

[10] D. A. Baker, M. A. Paesler, G. Lucovsky, S.C. Agarwal, and P.C. Taylor, Phys. Rev. Lett. 96, 255501 (2006).

[11] A. V. Kolobov, P. Fons, A. I. Frenkel, A.L. Ankudinov, J. Tominaga, and T. Uruga, Nat. 
Mater. 3, 703 (2004).

[12] S. Kohara, K. Kato, S. Kimura, H.U. Tanaka, T. Usuki, K. Suzuya, H. Tanaka, Y. Moritomo, T. Matsunaga, N. Yamada, Y. Tanaka, H. Suematsu, and M. Takata, Appl. Phys. Lett., 89, 201910 (2006).

[13] A. Fantini, V. Sousa, L. Perniola, E. Gourvest, J.C. Bastien, S. Maitrejean, S. Braga, N. Pashkov, A. Bastard, B. Hyot, A. Roule, A. Persico, H. Feldis, C. Jahan, J.F. Nodin, D. Blachier, A. Toffoli, G. Reimbold, F. Fillot, F. Pierre, R. Annunziata, D. Benshael, P. Mazoyer, C. Vallée, T. Billon, J. Hazart, B. De Salvo, and F. Boulanger, Proceedings of the IEEE International Electron Devices Meeting, 29.1.1 (2010).

[14] S. Caravati, D. Colleoni, R. Mazzarello, T.D. Kühne, M. Krack, M. Bernasconi, and M. Parrinello, J. Phys. Condens. Matter 23, 265801 (2011); and references therein.

[15] G.B. Beneventi, L. Perniola, A. Fantini, D. Blachier, A. Toffoli, E. Gourvest, S. Maitrejean, V. Sousa, C. Jahan, J.F. Nodin, A. Persico, S. Loubriat, A. Roule, S. Lhostis, H. Feldis, G. Reimbold, T. Billon, B. De Salvo, L. Larcher, P. Pavan, D. Bensahel, P. Mazoyer, R. Annunziata, and F. Boulanger, Proceedings of the European Solid-State Device Research Conference, $313(2010)$.

[16] G. B. Beneventi, L. Perniola, V. Sousa, E. Gourvest, S. Maitrejean, J.C. Bastien, A. Bastard, B. Hyot, A. Fargeix, C. Jahan, J.F. Nodin, A. Persico, A. Fantini, D. Blachier, A. Toffoli, S. Loubriat, A. Roule, S. Lhostis, H. Feldis, G. Reimbold, T. Billon, B. De Salvo, L. Larcher, P. Pavan, D. Bensahel, P. Mazoyer, R. Annunziata, P. Zuliani, and F. Boulanger, Solid-State Electronics 65-66, 197 (2011).

[17] G. Ghezzi, J.Y. Raty, S. Maitrejean, A. Roule, E. Elkaim, and F. Hippert, Appl. Phys.Lett. 99, 151906 (2011).

[18] A.V. Kolobov, P. Fons, B. Hyot, B. André, J. Tominaga, Y. Tamenori, H. Yoshikawa, and K. Kobayashi, Appl. Phys. Lett., 100, 061910 (2012).

[19] Y.M. Lee, H.J. Shin, S.J. Choi, J.H. Oh, H.S. Jeong, K. Kim, and M.-C. Jung, Current Appl. Phys. 11, 710 (2011).

[20] G. Naumis, and H.M. Flores-Ruiz, Phys. Rev. B 78, 094203 (2008).

[21] G. Baldi, A. Fontana, G. Monaco, L. Orsingher, S. Rols, F. Rossi, and B. Ruta, Phys. Rev. Lett. 102, 195502 (2009).

[22] A. I.Chumakov, G. Monaco, A. Monaco, W. Crichton, A. Bosak, R. Rüffer, A. Meyer, F. Kargl, 
L. Comez, D. Fioretto, H. Giefers, S. Roitsch, G. Wortmann, M. Manghnani, A. Hushur, Q. Williams, J. Balogh, K.. Parliński, P. Jochym, and P. Piekarz, Phys. Rev. Lett. 106, 225501 (2011).

[23] T. Rog, K. Murzyn, K. Hinsen, and G.R. Kneller, J. Comp. Chem. 5, 657 (2003).

[24] R. Shaltaf, E. Durgun, J.-Y. Raty, P. Ghosez, and X. Gonze, Phys. Rev. B 78, 205283 (2008).

[25] T. Matsunaga, N. Yamada, R. Kojima, S. Shamoto, M. Sato, H. Tanida, T. Uruga, S. Kohara, M. Takata, P. Zalden, G. Bruns, I. Sergueev, H.C. Wille, R.P. Hermann, and M. Wuttig, Adv. Fun. Mater. 21, 2232 (2011).

[26] $\mathrm{E}_{\mathrm{a}}$ is not directly accessible to the AIMD simulation as its calculation would require the direct simulation of the recrystallization process at various temperatures, with a statistical analysis and large box sizes.

[27] M. Micoulaut, J.-Y. Raty, C. Otjacques, and C. Bichara, Phys. Rev. B 81, 174206 (2010).

[28] H. M. Florez-Ruiz, G.G Naumis, and J.C. Phillips, Phys. Rev. B 82, 214201 (2010).

[29] H. M. Florez-Ruiz, and G.G. Naumis, J. Chem. Phys. 131, 154501 (2009).

[30] H. Florez-Ruiz, and G.G. Naumis, Phys. Rev. B 83, 184204 (2011).

[31] W. A. Kamitakahara, R. L. Cappelletti, P. Boolchand, B. Halfpap, F. Gompf, D. A. Neumann \& H. Mutka, Phys. Rev. B. 44, 94 (1991). 